\title{
3-D Model Supported Prostate Biopsy Simulation and Evaluation
}

\author{
Jianhua Xuan ${ }^{1}$ Yue Wang ${ }^{2,5}$ Isabell A. Sesterhenn ${ }^{3}$ \\ Judd W. Moul ${ }^{4}$ and Seong K. Mun ${ }^{5}$ \\ 1 University of Maryland Baltimore County, Baltimore 21250, USA \\ 2 The Catholic University of America, Washington, DC 20064, USA \\ 3 Armed Forces Institute of Pathology, Washington, DC 20306, USA \\ 4 Walter Reed Army Medical Center, Washinton, DC 20306, USA \\ ${ }^{5}$ Georgetwon University Medical Center, Washington, DC 20007, USA
}

\begin{abstract}
The diagnosis of localized prostate cancer is carried out by standard core needle biopsies under guidance of transrectal ultrasound imaging of the prostate gland. This paper describes a 3-D model supported virtual environment for prostate cancer diagnosis and biopsy design. A 3-D deformable reconstruction algorithm is developed to define object surface from digitally-imaged surgical specimens. A virtual environment with a multimodal visualization capability is integrated to simulate prostate biopsy protocols. The new system permits an accurate graphical modeling of the object of interest, the localization and quantification of tumors, and the definition of the pathways of biopsy needles. The technique allows the medical experts to probe and manipulate the data in 3-D view space and to evaluate the performance of simulated biopsies subsequently optimize biopsy techniques. Results and analysis of our experiments demonstrate the effectiveness of the individual modular components of the approach. We conclude with an application of the complete framework to a prostate biopsy simulation and evaluation task.
\end{abstract}

\section{Introduction}

Graphics-based surgical simulation and planning has phenomenal potential to improve medicine and the health care of mankind. With the advances of three dimensional computed imaging, the information obtained from digital images can be transformed into a computer-synthesized display that facilitates visualization of underlying disease patterns and spatial relationships. In particular, reconstructed 3-D computer models of human organs are used for surgery planning, simulation, image-guided therapy, and treatment assessment. We have advanced from rendering anatomy to aid diagnosis through interactive visualization of complicated or hidden anatomic structures and relationships to planning surgery and to simulate or assist surgery directly. New, more accurate and cost-effective surgical procedures have become possible by integrating computerized graphical models with medical images. For example, image-guided minimally invasive surgery are now streaming into clinical practice carrying medical visualization from diagnosis applications directly to patient care. 
The incidence of prostate cancer has increased over the past 10 years with approximately 317,000 American males being diagnosed with prostate cancer in 1996. Prostate cancer has overtaken lung cancer as the most prevalent malignancy in males and is now the second most common cause of cancer death in men. As a result of this rising incidence rate, it is of great interest to study early detection and treatment of this disease. Improved screening programs, prostatespecific antigen (PSA) radioimmunoassay, and increasing awareness of prostate cancer have resulted in a dramatically increased overall detection rate, particular for organ-confined tumors. However, due to the highly variable behavior of the prostate cancer and the lack of appropriate technologies, the complex disease patterns such as the volume, distribution, and multicentricity of the prostate cancer have not been incorporated into the staging and biopsy techniques. On the other hand, the treatment of localized prostate cancer has constantly advanced in surgical removal as well as the preservation of potency and continence, and radiation therapy over the past decade. Particularly, a number of new techniques in radiation therapy are being developed, such treatments as conformal radiation, proton therapy, combinations of radiation and hormone therapy, and radioactive seed implantation. It is of great interest in radioactive seed implantation to treat prostate cancer, since techniques of image-guided radiation therapy (ultrasound at present) can provide more accurate placements of these radioactive seeds within the prostate.

To increase early detection rate and deliver effective treatments of prostate cancer, we develop an image-guided biopsy simulation system with advanced image analysis and computer graphics techniques. Three-dimensional (3-D) computerized prostate models are reconstructed with an accurate 3-D representation of all internal anatomical structures of the prostate (e.g., urethra, seminal vesicles, ejaculatory ducts, etc.). The prostate needle biopsy simulation system is then implemented by an interactive $3-\mathrm{D}$ visualization system with various realistic imaging probes and needles for examination and path planning. With an accurate 3-D prostate model, realistic imaging probes and needles provided by our virtual simulation system, a surgeon can sit in front of the computer to plan better needle paths and further to practice the actual biopsy procedure before he/she actually performs on a patient. More importantly, by analyzing outcomes of this simulation, we can validate the effectiveness of various biopsy techniques in prostate cancer detection and tumor volume estimation.

\section{3-D Prostate Modeling}

Surgical prostate specimens from patients with biopsy-proven prostate cancer are sectioned into $4 \mu \mathrm{m}$ sections at $2.5 \mathrm{~mm}$ intervals, and each prostate specimen usually consists of $10-15$ such slices. All slices are then digitized by using a Leafscan 45 Scanner at a resolution of $1500 \mathrm{dpi}$. Slices of each prostate specimen are aligned by a feature-based manual registration performed by pathologists using Photoshop. Finally digital images are stored on CDROMs as our prostate database. Contour extraction and segmentation are conducted manually 
by pathologists on Photoshop guided by microscopic section information. The regions of interest are the prostate capsule, surgical margins, urethra, seminal vesicles, ejaculatory ducts, carcinomas, and all areas of PIN .

Reconstruction of the prostate model is achieved by using a new deformable surface-spine model described as follows: A deformable spine (axis) of the prostate model is determined from its contours, then all the triangular finite elements are contracted to the spine through expansion/compression forces radiating from the spine while the spine itself is also confined to the surfaces. The surface refinement is governed by a second-order partial differential equation from Lagrangian mechanics, and the refining process terminates when the energy of this dynamic deformable surface-spine model reaches its minimum [2]. Figure 1(a) shows a complete reconstructed 3 -D prostate model rendered with all anatomical structures such as the prostate capsule, urethra, seminal vesicles, ejaculatory ducts and carcinomas.

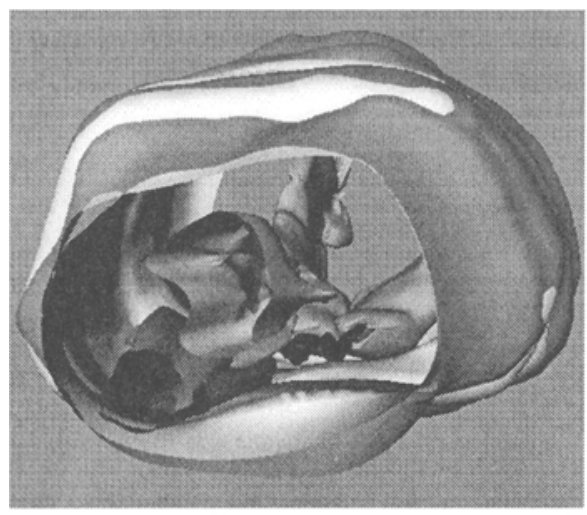

(a)

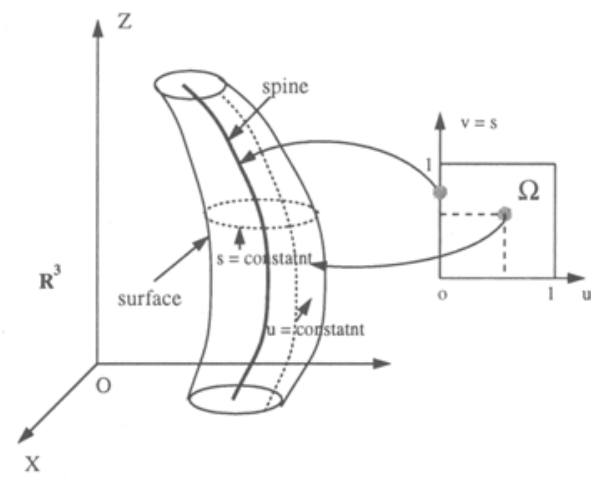

(b)

Fig. 1. (a) Reconstructed prostate model. (b) Mathematical model.

We define the surface and spine as geometric mappings from material (parametric) coordinate domains into three-dimensional (3-D) Euclidean space $\Re^{3}$. The strain energy $\mathcal{E}$ can be found to characterize the deformable material of either the surface or spine, which will be discussed in the next section as an instance of the spline function. Then the continuum mechanical equation

$$
\mu \frac{\partial^{2} \mathbf{x}}{\partial t^{2}}+\gamma \frac{\partial \mathbf{x}}{\partial t}+\frac{\delta \mathcal{E}(\mathbf{x})}{\delta \mathbf{x}}=\mathbf{f}(\mathbf{x}),
$$

governs the non-rigid motion of the surface (spine) in response to an extrinsic force $\mathbf{f}(\mathbf{x})$, where $\mu$ is the mass density function of the deformable surface (spine) and $\gamma$ is the viscosity function of the ambient medium. The third term on the 
left-hand side of the equation is the variational derivative of the strain energy functional $\mathcal{E}$, the internal elastic force of the surface (spine).

The deformable energy of surface $\mathbf{x}(u, v, t)$ can be definned by

$$
\begin{aligned}
\mathcal{E}_{\text {surface }}(u, v, t)=\int_{0}^{1} & \int_{0}^{1}\left(w_{10}\left|\frac{\partial \mathbf{x}}{\partial u}\right|^{2}+2 w_{11}\left|\frac{\partial \mathbf{x}}{\partial u}\right| \times\left|\frac{\partial \mathbf{x}}{\partial v}\right|+w_{01}\left|\frac{\partial \mathbf{x}}{\partial v}\right|^{2}\right. \\
& \left.+w_{20}\left|\frac{\partial^{2} \mathbf{x}}{\partial u^{2}}\right|^{2}+2 w_{22}\left|\frac{\partial^{2} \mathbf{x}}{\partial u \partial v}\right|^{2}+w_{02}\left|\frac{\partial^{2} \mathbf{x}}{\partial v^{2}}\right|^{2}\right) d u d v,(2)
\end{aligned}
$$

where the weights $w_{10}, w_{11}$ and $w_{11}$ control the tensions of the surface, while $w_{20}, w_{22}$ and $w_{02}$ control its rigidities (bending energy). The deformable energy of spine $\mathbf{x}(u, t)$ is given by

$$
\mathcal{E}_{\text {spine }}(s, t)=\int_{0}^{1}\left(w_{1}\left|\frac{d \mathbf{x}}{d s}\right|^{2}+w_{2}\left|\frac{d^{2} \mathbf{x}}{d s^{2}}\right|^{2}\right) d s .
$$

The weight $w_{1}$ controls the tension along the spine (stretching energy), while $w_{2}$ controls its rigidity (bending energy).

To couple the surface with the spine, we enforce $v \equiv s$, which maps the spine coordinate into the coordinate along the length of the surface as shown in Figure 1(b). Then we connect the spine with surface by introducing following forces on the surface and spine respectively [3]:

$$
\begin{aligned}
& \mathbf{f}_{\text {surface }}^{a}(u, s, t)=-(a / l)\left(\overline{\mathbf{x}}_{\text {surface }}-\mathbf{x}_{\text {spine }}\right) \\
& \mathbf{f}_{\text {spine }}^{a}(s, t)=a\left(\overline{\mathbf{x}}_{\text {surface }}-\mathbf{x}_{\text {spine }}\right)
\end{aligned}
$$

where $a$ controls the strength of the forces; $\overline{\mathbf{x}}_{\text {surface }}$ is the centroid of the coordinate curve $\left(s=\right.$ constant) circling the surface and defined as $\overline{\mathbf{x}}_{\text {surface }}=$ $\frac{1}{l} \int_{0}^{1} \mathbf{x}_{\text {surface }}\left|\frac{\partial \mathbf{X}_{s u r f a c e}}{\partial u}\right| d u$, where $l$ is the length given by $l=\int_{0}^{1}\left|\frac{\partial \mathbf{X}_{\text {surface }}}{\partial u}\right| d u$. In general, the above forces coerce the spine staying on an axial position of the surface. Further, if necessary, we can encourage the surface to be radially symmetric around the spine by introducing the following force:

$$
\mathbf{f}_{\text {surface }}^{b}=b(\overline{\mathbf{r}}-|\mathbf{r}|) \hat{\mathbf{r}},
$$

where $b$ controls the strength of the force; $\mathbf{r}$ is the radial vector of the surface with respect to the spine as $\mathbf{r}(u, s)=\mathbf{x}_{\text {surface }}-\mathbf{x}_{\text {spine }}$, the unit radial vector $\hat{\boldsymbol{r}}(u, s)=$ $\mathbf{r} /|\mathbf{r}|$, and $\overline{\mathbf{r}}(s)=\frac{1}{l} \int_{0}^{1}|\mathbf{r}| \frac{\partial \mathbf{X}_{s u r f a c e}}{\partial u} d u$, as the mean radius of the coordinate curve $s=$ constant. Also, it is possible to provide control over expansion and contraction of the surface around the spine. This can be realized by introducing the following force:

$$
\mathbf{f}_{\text {surface }}^{c}=c \hat{\mathbf{r}},
$$

where $c$ controls the strength of the expansion or contraction force. The surface will inflate where $c>0$ and deflate where $c<0$. 
Summing the above coupling forces in the motion equation associated with surface and spine, we obtain the following dynamic system describing the motion of the deformable surface-spine model:

$$
\begin{array}{r} 
\\
\mu \frac{\partial^{2} \mathbf{x}_{\text {surface }}}{\partial t^{2}}+\gamma \frac{\partial \mathbf{x}_{\text {surface }}}{\partial t}+\frac{\delta \mathcal{E}_{\text {surface }}}{\delta \mathbf{x}} \\
=\mathbf{f}_{\text {surface }}^{\text {ext }}+\mathbf{f}_{\text {surface }}^{a}+\mathbf{f}_{\text {surface }}^{b}+\mathbf{f}_{\text {surface }}^{c} \\
\mu \frac{\partial^{2} \mathbf{x}_{\text {spine }}}{\partial t^{2}}+\gamma \frac{\partial \mathbf{x}_{\text {spine }}}{\partial t}+\frac{\delta \mathcal{E}_{\text {spine }}}{\delta \mathbf{x}}=\mathbf{f}_{\text {spine }}^{\text {ext }}+\mathbf{f}_{\text {spine }}^{a},
\end{array}
$$

where $\mathbf{f}_{\text {surface }}^{e x t}$ is the external force applied on the surface and $\mathbf{f}_{\text {spine }}^{\text {ext }}$ the external force applied on the spine.

Both the finite difference method and the finite element method can be used to compute the numerical solution to the surface $\mathbf{x}_{\text {surface }}$ and spine $\mathbf{x}_{\text {spine }}$. Finite difference method approximates the continuous function $\mathbf{x}$ as a set of discrete nodes in space. A disadvantage of the finite difference approach is that the continuity of the solution between nodes is not made explicitly. The finite element method, on the other hand, provides continuous surface (or spine) approximation by approximating the unknown function $\mathbf{x}$ in terms of combinations of the basis functions. In finite element method, we first tessellate the continuous material domain, $(u, v)$ for the surface and $s$ for the spine in our case, into a mesh of $m$ element subdomains $D_{j}$, then we approximate $\mathbf{x}$ as a weighted sum of continuous basis functions $\mathbf{N}_{i}$ (so-called shape functions): $\mathbf{x} \approx \mathbf{x}^{h}=\sum_{i} \mathbf{x}_{i} \mathbf{N}_{i}$, where $\mathbf{x}_{i}$ is a vector of nodal variables associated with mesh node $i$. The shape functions $\mathbf{N}_{i}$ are fixed in advance and the nodal variables $\mathbf{x}_{i}$ are the unknowns. The motion equation can then be descritized as

$$
\mathbf{M} \frac{\partial^{2} \mathbf{x}}{\partial t^{2}}+\mathbf{C} \frac{\partial \mathbf{x}}{\partial t}+\mathbf{K} \mathbf{x}=\mathbf{F},
$$

where $\mathbf{x}=\left[\mathbf{x}_{1}^{T}, \ldots, \mathbf{x}_{i}^{T}, \ldots, \mathbf{x}_{n}^{T}\right], \mathbf{M}$ is the mass matrix, $\mathbf{C}$ the damping matrix, $\mathbf{K}$ the stiff matrix, and $\mathbf{F}$ the forcing matrix. $\mathbf{M}, \mathbf{C}$, and $\mathbf{F}$ can be obtained as follows:

$$
\mathbf{M}_{\mathbf{j}}=\iint_{E_{j}} \mu \mathbf{N}_{j}^{T} \mathbf{N}_{j} d u d v, \quad \mathbf{C}_{\mathbf{j}}=\iint_{E_{j}} \gamma \mathbf{N}_{j}^{T} \mathbf{N}_{j} d u d v, \quad \mathbf{F}_{\mathbf{j}}=\iint_{E_{j}} \mathbf{N}_{j}^{T} \mathbf{f}_{j} d u d v
$$

To compute $\mathbf{K}$, we have the following equation:

$$
\mathbf{K}_{j}=\iint_{E_{j}}\left(\mathbf{N}_{b}^{T} \beta \mathbf{N}_{b}+\mathbf{N}_{s}^{T} \alpha \mathbf{N}_{s}\right) d u d v, \alpha=\left[\begin{array}{cc}
w_{02} & w_{22} \\
w_{22} & w_{20}
\end{array}\right], \beta=\left[\begin{array}{ccc}
w_{01} & 0 & 0 \\
0 & w_{11} & 0 \\
0 & 0 & w_{10}
\end{array}\right]
$$

where $\mathbf{N}_{b}=\left[\frac{\partial^{2} \mathbf{N}}{\partial u^{2}}, \frac{\partial^{2} \mathbf{N}}{\partial u \partial v}, \frac{\partial^{2} \mathbf{N}}{\partial v^{2}}\right]^{T}, \mathbf{N}_{s}=\left[\frac{\partial \mathbf{N}}{\partial u}, \frac{\partial \mathbf{N}}{\partial v}\right]^{T}$. 


\section{The Deformable Surface Element}

The deformable surface consists of a set of connected traingular elements chosen for their capability to model a large range of topological shapes. Barycentric coordinates in two dimensions are the natural choice for defining shape functions over a triangular domain. Barycentric coordinates $\left(L_{1}, L_{2}, L_{3}\right)$ are defined by the following mapping with material coordinates $(u, v)$ :

$$
\left[\begin{array}{l}
u \\
v \\
1
\end{array}\right]=\left[\begin{array}{ccc}
u_{1} & u_{2} & u_{3} \\
v_{1} & v_{2} & v_{3} \\
1 & 1 & 1
\end{array}\right]\left[\begin{array}{l}
L_{1} \\
L_{2} \\
L_{3}
\end{array}\right]
$$

where $\left(u_{1}, v_{1}\right),\left(u_{2}, v_{2}\right)$, and $\left(u_{3}, v_{3}\right)$ are the coordinates of three vertex locations of the triangle.

We use the 9 degree-of-freedom (dof) triangular element which includes the position and its first parametric partial derivatives at each triangle vertex. The shape functions of the first node in a 9 dof triangle are [4]:

$$
\mathbf{N}_{1}^{9^{T}}=\left[\begin{array}{c}
N_{1} \\
N_{2} \\
N_{3}
\end{array}\right]=\left[\begin{array}{c}
L_{1}+L_{1}^{2} L_{2}+L_{1}^{2} L_{3}-L_{1} L_{2}^{2}-L_{1} L_{3}^{2} \\
c_{3}\left(L_{1}^{2} L_{2}+0.5 L_{1} L_{2} L_{3}\right)-c_{2}\left(L_{1}^{2} L_{3}+0.5 L_{1} L_{2} L_{3}\right) \\
-b_{3}\left(L_{1}^{2} L_{2}+0.5 L_{1} L_{2} L_{3}\right)+b_{2}\left(L_{1}^{2} L_{3}+0.5 L_{1} L_{2} L_{3}\right)
\end{array}\right] .
$$

The triangle's symmetry in Barycentric coordinates can be used to generate the shape function for the second and third nodes in terms of the first. To generate $\mathrm{N}_{2}^{9}$ use the above equations but add a 1 to each index so that $1 \rightarrow 2,2 \rightarrow 3$ and $3 \rightarrow 1$. The $\mathbf{N}_{3}^{9}$ functions can be obtained by adding another 1 to each index. Note that the shape functions for 9 dof triangle do not guarantee $C^{1}$ continuity between adjacent triangular elements. In [4], a 12 dof triangular element can be made $C^{1}$ continuous by adding a 1 dof on each edge of the triangle, see [5] for the detail. An alternative to have $C^{1}$ continuous triangular element is to use a 18 dof element which include the nodal location, its first and second partial derivatives evaluated at each node [6]. In this paper we use 9 dof triangular element although the extension to 12 or 18 dof triangular element is straight-forward.

\section{The Deformable Spine Element}

The finite element of the spine has 4 dofs between two nodes located at the ends of the segment. The dofs at each node correspond to its position and tangent. The spine segment can be approximated as the weighted sum of a set of Hermite polynomials: $\mathbf{x} \approx \mathbf{x}^{h}(s)=\sum_{i=0}^{3} \mathbf{x}_{i} N_{i}$, where $N_{i}, i=0, \ldots, 3$ are given as follows:

$$
\begin{aligned}
& N_{0}=1-3(s / h)^{2}+2(s / h)^{3}, \\
& N_{1}=h\left(s / h-2(s / h)^{2}+(s / h)^{3}\right), \\
& N_{2}=3(s / h)^{2}-2(s / h)^{3}, \\
& N_{3}=h\left(-(s / h)^{2}+(s / h)^{3}\right),
\end{aligned}
$$

where $h$ is the parametric element length. 
3 -D prostate models can be interactively visualized by the state-of-the-art graphics toolkit, object-oriented OpenInventor. With a sophisticated set of various kinds of lights, 3-D manipulators, and color and material editors, etc., we can examine the 3-D prostate model in any viewpoint and interactively walk through it to better understand relationships of anatomical structures of the prostate and its tumors.

\section{$3 \quad$ Needle Biopsy Simulation}

The diagnosis of both the clinically palpable and the non-palpable prostatic lesion requires tissue diagnosis. Biopsy is required in all patients with suspected prostatic malignancy. Conventional techniques are usually performed with digital rectal guidance. The use of digital biopsy can only be performed when a palpable area of abnormality is identified. However, with small lesions, this is not an accurate technique. Thus, for diagnosis of non-palpable lesions or of small and subtly palpable ones, ultrasound guided biopsy can be performed accurately, safely and quickly. Transperineal biopsy and transrectal biopsy are two common approaches that can be utilized in the prostate cancer diagnosis. Fig. 2(a) shows the diagram of transrectal biopsy whose procedure will be simulated. Under ultrasound guidance, the needle is placed through the guide into the lesion (Fig. $2(\mathrm{~b}))$.

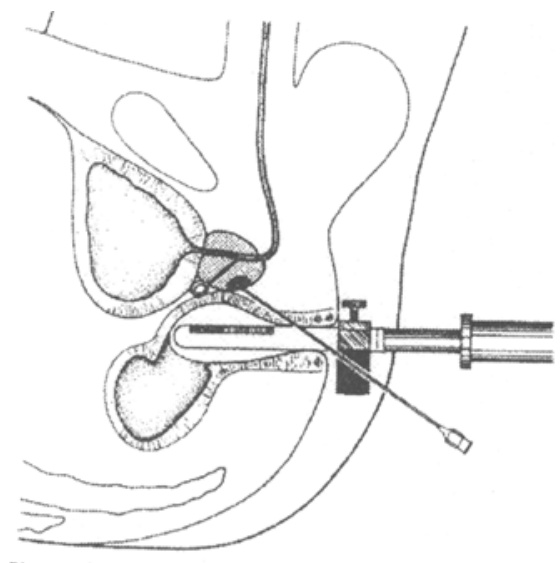

(a)

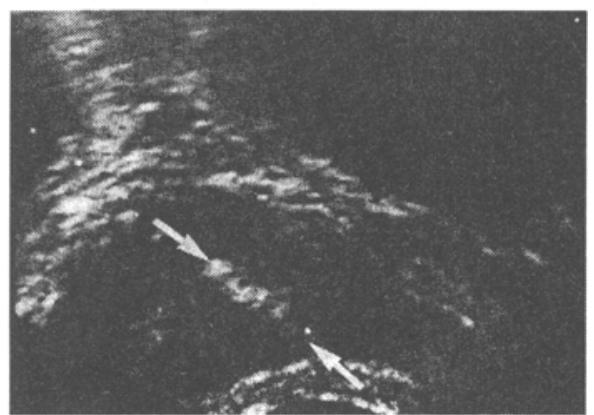

(b)

Fig. 2. (a) Diagram of the prostate needle biopsy, (b) the ultrasonic image.

In this section, we first describe an image-guided needle biopsy simulation system implemented with a real-time imaging probe and a needle tracking system. Then we implement a systematic needle biopsy simulation system which 
enables us to validate various blind biopsy techniques by comparing their cancer detection rates.

\subsection{Image-Guided Needle Biopsy Simulation}

The image-guided needle biopsy simulation system simulate the clinical procedures of prostate cancer biopsy in the following two steps:

(i) Examining the prostate using the ultrasound-like probe: Different ultrasoundlike imaging probes are simulated to provide axially and/or longitudinally oriented sectional images for efficiently planning needle pathways.

(ii) Defining the needle paths and performing simulated biopsy: Needles with or without triggers are constructed and simulated to perform actual biopsy on 3-D computerized prostate models according to the planned needle pathways, see Figure 3 (a).

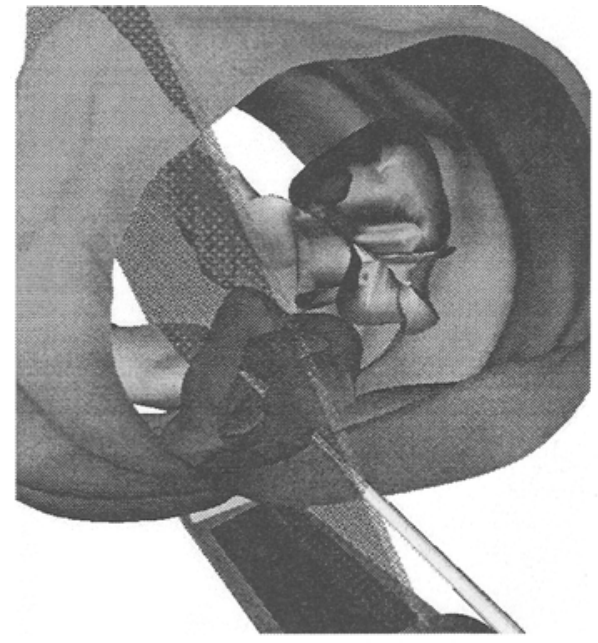

(a)

\begin{tabular}{||c|c|c|c||}
\hline & SRSCB & S5RB & SRSCB+S5RB \\
\hline \hline T1c (24\%) & $10(77 \%)$ & $9(69 \%)$ & $10(77 \%)$ \\
\hline T2 (76\%) & $37(88 \%)$ & $33(79 \%)$ & $38(90 \%)$ \\
\hline
\end{tabular}

(b)

Fig. 3. (a) 3-D view of the needle biopsy simulation, (b) Clinical stage and positive biopsy distribution.

\subsection{Systematic Needle Biopsy Simulation}

In 1989, Hodge et al. proposed the technique of six random systematic core biopsy (SRSCB) of the prostate gland. The technique has gained acceptance by urologists for detection of carcinoma of the prostate $(\mathrm{CaP})$ since then. With the image-guided needle biopsy simulation system we can verify the validity 
of the SRSCB technique in detecting prostate cancer and possibly recommend new biopsy technique to increase the detection rate. We have implemented both SRSCB and systematic 5-region biopsy technique (S5RB) in our simulation system.

In this simulation, we have reconstructed 55 computerized prostate models of prostate specimens taken from patients and performed biopsy techniques on them. The simulation results are shown in Figs. 4 and 5, where the detection probability of each needle can be calculated to indicate the clinical importance. Fig. 3 (b) gives the clinical stage and positive biopsy in 55 patients, where T1c means small tumor and T2 means large tumor. By correlating the simulation results with clinical data such as PSA we can evaluate the biopsy strategies for detection of prostate cancer. In addition, the prostate biopsy simulation system can show the biopsy procedure in 3-D space to record and analyze various causes of hit or miss in individual cases.

\begin{tabular}{|l|c|c||}
\hline & \# of Positive Cores & \% of Total Cores \\
\hline \hline L-Base & 13 & $10 \%$ \\
\hline L-Mid & 23 & $17 \%$ \\
\hline L-Apex & 27 & $20 \%$ \\
\hline R-Base & 7 & $5 \%$ \\
\hline R-Mid & 29 & $22 \%$ \\
\hline R-Apex & 34 & $26 \%$ \\
\hline Total & 133 & \\
\hline
\end{tabular}

(a)
Positive Biopsy Distribution (SRSCB)

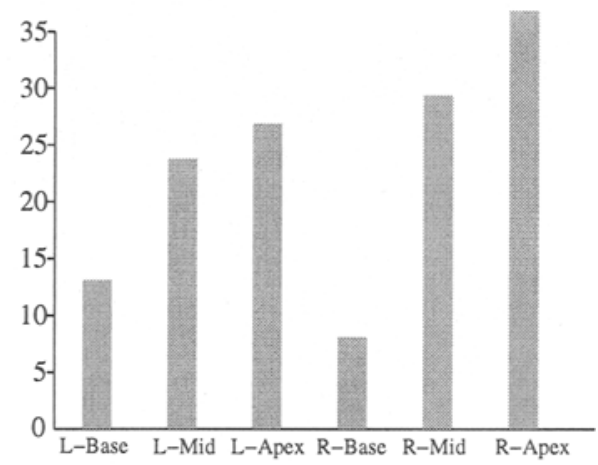

(b)

Fig. 4. Positive biopsy distribution (SRSCB): (a) table, (b) histogram.

\section{Conclusion}

In this paper, we have developed a vitual environment for prostate needle biopsy simulation using advanced image analysis and computer graphics techniques. 3D computerized prostate models are first reconstructed from microscopic images of surgical prostate specimens. Computer simulation systems are then implemented to simulate both image-guided and systematic prostate needle biopsy 


\begin{tabular}{|l|c|c||}
\hline & \# of Positive Cores & $\%$ of Total Cores \\
\hline \hline L-Base & 12 & $12 \%$ \\
\hline L-Apex & 14 & $14 \%$ \\
\hline M-Base & 2 & $2 \%$ \\
\hline M-Apex & 30 & $31 \%$ \\
\hline R-Base & 13 & $13 \%$ \\
\hline R-Apex & 26 & $27 \%$ \\
\hline Total & 97 & \\
\hline
\end{tabular}

(a)
Positive Biopsy Distribution (S5RB)

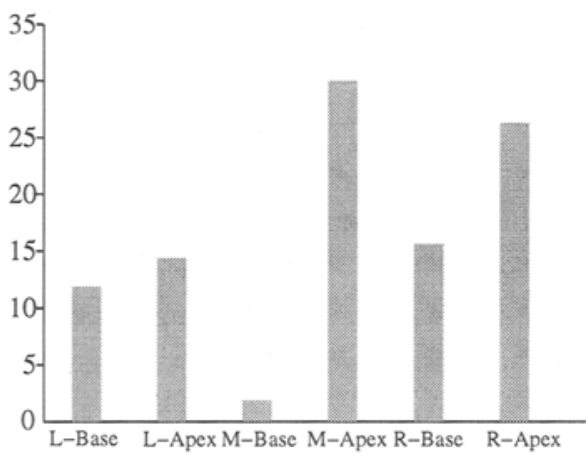

(b)

Fig. 5, Positive biopsy distribution (S5RB): (a) table, (b) histogram.

techniques with interactive visualization and manipulation capabilities. With such a simulation environment, a surgeon can better plan needle paths in 3-D space in order to increase cancer detection rate. Simulation results on various prostate needle biopsy techniques have also demonstrated that we can validate the effectiveness of different biopsy techniques in prostate cancer detection by analyzing the simulation outcomes.

\section{References}

1. A. Chiarodo, "National Cancer Institute roundtable on prostate cancer: Future directions," Cancer Res. vol. 51, pp. 2498-2505, 1991.

2. J. Xuan, I. Sesterhenn, W. Hayes, Y. Wang, T. Adali, M. Freedman, and S. K. Mun, "Surface reconstruction and visualization of surgical prostate models," Medical Imaging, Newport Beach, Feb. 1997.

3. D. Terzopoulos, A. Witkin, and M. Kass, "Symmetry-seeking models and 3D object reconstruction," Int. J. Computer Vision, vol. 1, pp. 211-221, 1987.

4. Zienkiewicz, The Finite Element Method, The Third Edition, McGraw-Hill Book, 1967.

5. G. Celniker and D. Gossard, "Deformable curve and surface finite-elements for freeform shape design," Computer Graphics, Vol. 25, No. 4, pp. 257-266, July 1991.

6. T. McInerney, and D. Terzopoulos, "A dynamic finite element surface model for segmentation and tracking in multidimensional medical images with application to cardiac 4D image analysis," Computerized Medical Imaging and Graphics, vol. 19, no. 1, pp. 69-83, 1995. 